\title{
AN IMPROVED ENERGY-EFFICIENT HYBRID FRAMEWORK EEHF - ALGORITHM FOR GREEN CLOUD COMPUTING
}

\author{
Mrs. Sangeetha \\ Student, Department of Computer Science and Engineering, P. S. R. Engineering College, \\ Sivakasi, 626140, Tamil Nadu, India \\ R. Arun \\ Assistant Professor, Department of Computer Science and Engineering, P. S. R. Engineering College, \\ Sivakasi, 626140, Tamil Nadu, India
}

\begin{abstract}
This paper deals with the relocation of multi-target Virtual Machines (VMs) in a cloud server farm. The proposed VM movement technique at the cloud server farm meanders VMs from underutilized to full capacity Physical Machines (PMs) to energy-efficient Physical Machines (PMs). Furthermore, the multitarget VMs relocation technique not only reduces the forced use of PMs and switches, but also confirms the essence of administration by preserving the SLA at the cloud server farm. A novel energy-efficient hybrid (EEHF) system for enhancing the proficiency of electrical energy usage in data centres is carried out and evaluated in this paper. Instead of focusing on only one approach as in previous related works, the proposed system is truly based on solicitation preparation and worker booking. Until managing the preparation, the EEH system sorts the errand clients' requests according to their time and force requirements. It has a booking system that takes power use into account when making planning decisions. It also has a precise calculation that determines if under burdened employees should be rested or dozed in overburdened workers, virtual machines that should be floated, and workers that will receive moved virtual machines VMs. When compared to other strategies, our proposed VMs development strategy may find a great balance among three conflict goals. Furthermore, the shroud-based cloud sim test results show that our proposed multi-target VMs relocation strategy outperforms best-in-class VMs movement strategies like the Random VMs relocation system in terms of energy efficiency and SLA penetration at the cloud server farm.
\end{abstract}

Keywords: Cloud Computing; Data Centre; Energy Efficiency; Virtual Machine.

\section{Introduction}

Virtualization technology is currently being used in server farms to convert the stock of a single PM into a few disengaged execution environments expressed on VMs. As a result, the primary benefit of this strategy is fewer numbers of PMs with much higher per-PM usage, increasing adaptability and usability, lowering equipment costs, and other operating costs such as force, cooling, physical split, and so on, even though virtualization's upsides carry a lot of face before information centre costs, such as assignment and the executives of virtualized properties. Furthermore, as a result of the central management of cloud benefits, the cloud server farm's energy consumption is gradually increasing.

IT devices consume 45 percent of the total energy consumed by cloud server farms [1]. Staff, switches, switches, interconnection joins, and other IT devices are included in the IT gadgets. Furthermore, organization components such as connectors, switches, and collection components account for 33\% of total energy consumption. The staff at the cloud server farm used an additional 66 percent of the total energy available. Other components of the cloud server farm, such as force and cooling conveyance modules, consume $15 \%$ and 45 percent of the total energy consumption, respectively. Furthermore, there are a variety of strategies available to reduce the cloud server farm's energy consumption. At the cloud server farm, for example, energy-efficient VMs are designated, tasks are booked, and movement is performed using environmentally friendly power.

There aren't many current works for reducing worker energy consumption by prioritizing the use of energyefficient VMs in cloud server farms [1], [3], [4], [5]. However, these studies did not evaluate the organization's 
energy use or the movement of energy-efficient VMs at the cloud server farm. Furthermore, a primary boundary such as QoS is needed to reduce the cloud server farm's energy consumption. As a result, the current review assumes that a disproportionately small amount of work has been completed in terms of multi-target VMs relocation at the cloud server farm. This led us to propose the multi-target VMs strategy, which not only reduces the energy consumption of PMs and switches but also avoids SLA violations at the cloud server farm. The main idea of our proposed work in this paper was to distribute the clients' request VMs at the information using the First Fit guess technique, and then to transfer the VMs from PMs using our set Energy-Efficient Hybrid Framework (EEHF) gauge dependent VMs movement strategy. to the cloud server farm's energy-efficient PMs, and turning off inactive PMs and switches in the cloud server farm. As a result, our completed work reduces energy consumption while also avoiding SLA violations at the cloud server farm. This paper's vital assistance is as follows:

1) To create a mathematical model of the cloud server farm's multi-target VM entry problem.

2) To set up and develop an EEHF guess technique-based VM relocation practice that not only reduces the energy consumption of PMs and switches but also avoids SLA violations at the cloud server farm.

3) To monitor energy consumption and asset usage in a cloud server farm with a heterogeneous collection of environmental factors.

The remainder of this piece of paper is color-coordinated as follows. The second segment discusses the foundation and related work of the multi-target VMs relocation problem. The third section deals with the planned work on the multi-target VMs relocation problem. Section 4 follows, arranging the results and analysis. Finally, Section 5 concludes with the paper containing future bearings.

\section{Background and Related Works}

The board strategy previously dealt with energy-efficient assets and VMs at the cloud server farm was partitioned into two fundamental classifications: single-target and multi-target Virtual Machines (VMs).

Management of single virtual machines (VMs) C. H. Hsu [7] devised and constructed a self-contained Voltage Frequency Scaling (SVFS) support single force saving technique for a cloud server farm worker. In their work, creators reduce a worker's force use by restricting CPU use with high edge esteem. As a result, the developers modified the VMs' working recurrence based on the actual responsibility of the VMs in their methodology. However, because the maker rejected the numerous Virtual Machine PMs Power-saving strategies, C. M. Wu et al. [8] suggested an energy-efficient errand booking enhancer. Regardless, the developers did not use the cloud server farm's energy-efficient Virtual Machine VMs allocation.

Virtual Machine VMs Management with Several Targets is a job title. Lee et al. [9] implemented a constructive cross-layer protocol for programmed the cloud server farm executives to restrict the use of warm heat and nuclear power at the cloud server farm. Furthermore, the developers failed to account for the shared Virtual Machine VMs at the cloud server farm. M. Polverini et al. [10] devised an online multi-target transition job planning technique for the circulated cloud server farms to resolve the issue of having data about the prior status of cloud server farms. Creators met the energy cost, lining challenge, and warm temperature of cloud server farms in their work. Furthermore, according to the role planning strategy, occupations progress when the line range is agreeably large or the power costs are appropriate. Their work is limited because he did not consider energy-conscious Virtual Machine VMs allocation at the cloud server farm.

\section{Proposed Work}

\subsection{Power Consumption Method}

Allow us to acknowledge the PM's CPU use at the time 't', which is denoted by (ut). Furthermore, (P min) and (P max) separately discuss the PM's minimum and most extreme force consumption. Eq. 1 depicts the force utilization of a PM in this way.

$$
P_{j}=\left(\left[P_{j}^{\max }-P_{j}^{\text {min }}\right]\right) u+P_{j}^{\text {min }}
$$

Furthermore, the server farm's force utilization of a switch is dependent on the flow of traffic through the device and its equipment design, such as (processor board, memory, cooling framework, and so on) [18]. As a result, we can reduce switch power consumption in the following ways [19], [20]: 
I) A single switch port saves 0.8 percent of a switch's total power consumption; ii) A line card saves the most absolute force usage (37 percent). iii) Setting a port to a lower transmission frequency (from 100 Mbps to 1 Gbps) saves between 0.05 and 0.19 percent of a switch's total power consumption. As a result, Eq. 2 depicts the force utilization of an organization switch.:

$$
P_{s}^{\text {switch }}(t)=P_{s}^{\text {idle }}+P_{s}^{\text {port }} * n(p)
$$

To reduce the switch's force consumption, we must kill the line card or switch's inactive ports in this manner. Consider the following: a group of switches at a server farm is denoted by $\mathrm{S}$, and a specific switch is denoted by $\mathrm{s}$, as in $\mathrm{S} \mathrm{S}$.

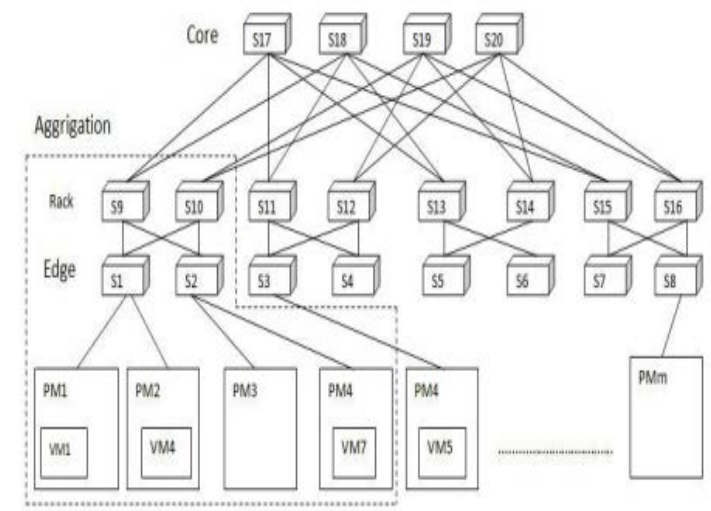

Fig. 1. Fat-tree Architecture of Data Centre

The PMs will be harmed by the allocated Virtual Machine VMs listed by the clients until their life has ended. As a result of the annihilation of Virtual Machine VMs from PMs, the specialist co-op will be able to float VMs from underutilized PMs to more energy-efficient PMs and then switching off unused PMs and switches at the cloud server farm. As a result, the goal ability for multi-target Virtual Machine VMs relocation is unique in that Virtual Machine VMs are linked from one Source node (PM) to a Destination node (PM). To the point where a larger number of VMs are linked to the destination node within their power. After the VMs from the source have been associated, we switch off the source node at the server farm. As a result, the cloud server farm has the largest number of unused PMs in the killed state. Furthermore, the SLA penalty for Virtual Machine VMs power-off time is the same as the Virtual Machine VMs lease, which the consumer can pay during the power-off period. The ward on data transfer capability of the system, the amount of memory substance to be replicated from the source node PM to the destination node PM, and so forth. As a result, we were able to apply a fixed force utilization of $\mathrm{Pk}$ to enormous, medium, small, and $\mathrm{x}$-huge as a movement overhead number in our research. Following that, we deal with all the Virtual Machine VMs assembled by the destination nodes in the expanding request based on their resource requirement and Virtual Machine VMs in the diminishing request based on their left side over resources limit, and then we revamp the Virtual Machine VMs from unused PMs to energy-efficient PMs using the First Fit Decreasing strategy, and along the way.

\section{Results and Analysis}

We used a cloud sim simulator to verify the display of the planned multi-target Virtual Machine VMs component strategy in a network-aware cloud server farm scenario. The cloud sim simulator simulates an organization's cloud server farm, and we can test our Virtual Machine VMs relocation rule within the simulator to validate cloud server farm best practices. Figure 2 shows a complete representation of the clients listed Virtual Machines VMs. In addition, the VMs' life season is depicted in Fig. 3. Figures 4 and 5 depict the energy consumption and normal resource usage at the cloud server farm, respectively. The amount of energy saved by clients when using the proposed EEHF has been discovered, according to ECTC and PRS. The x-pivot displays the number of client requests, while the y-hub addresses the amount of energy saved. In comparison to the other calculations, the proposed EEHF structure has the highest degree of energy savings, as seen in the graph. This is because, unlike other estimates, the EEHF system uses two procedures for negligible use of electrical force rather than depending on a single technique. In comparison to other methods, the EEHF aims to use a small number of resources in virtual machines. As a result, several Virtual Machines may be available to serve various solicitations or activities. As a result, the EEH Framework saves significantly more money than the other algorithms. 


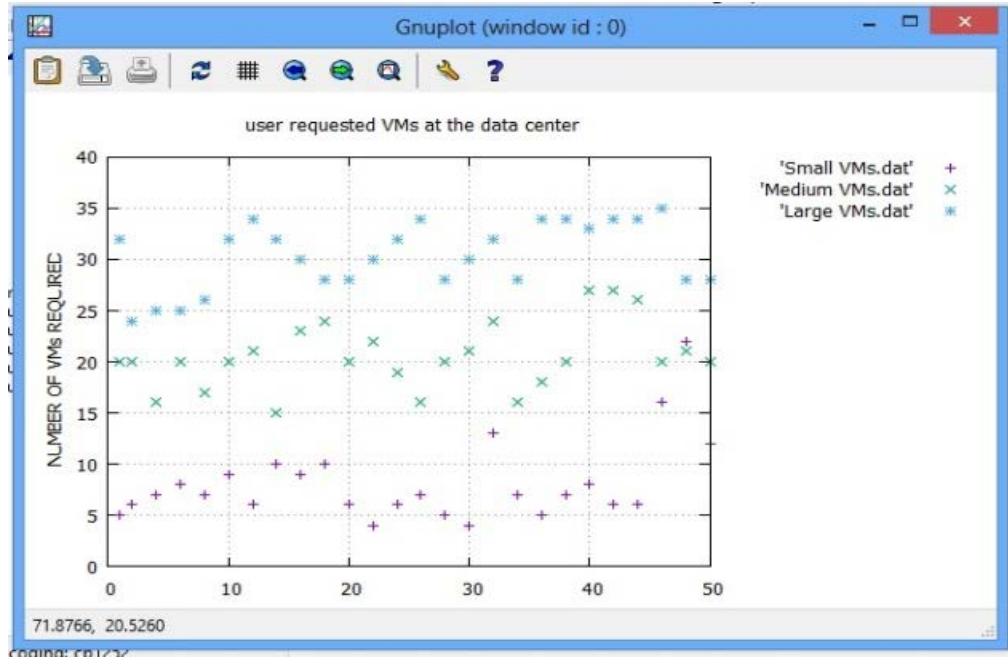

Fig. 2. Users Requested VMs at the Data Center

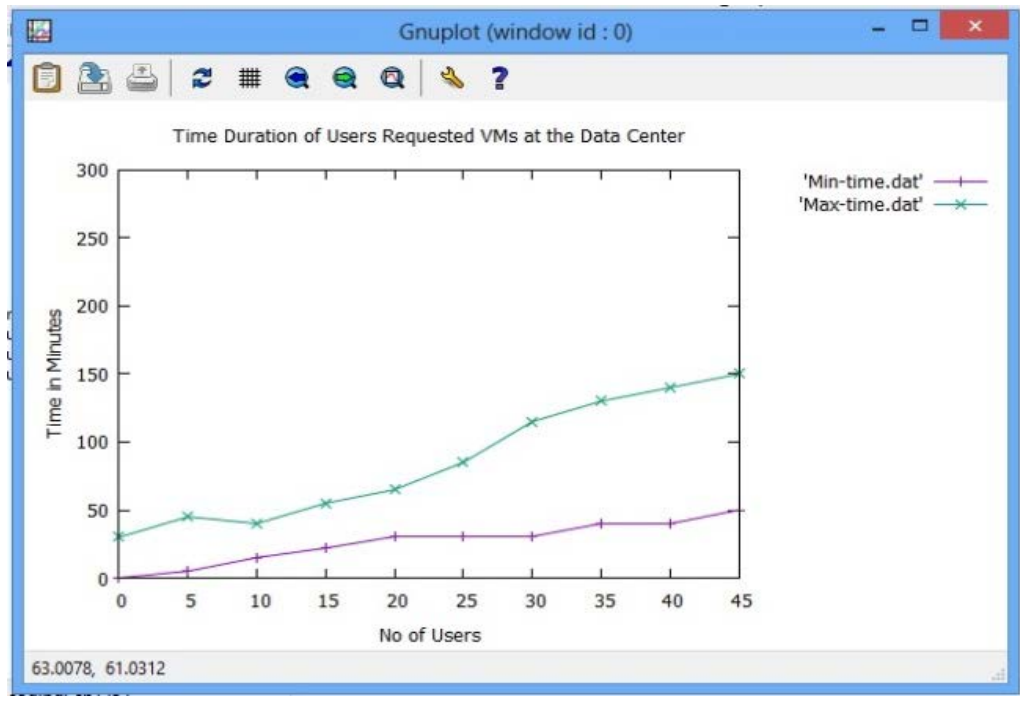

Fig. 3. Time Duration of Users Requested VMs at the Data Center

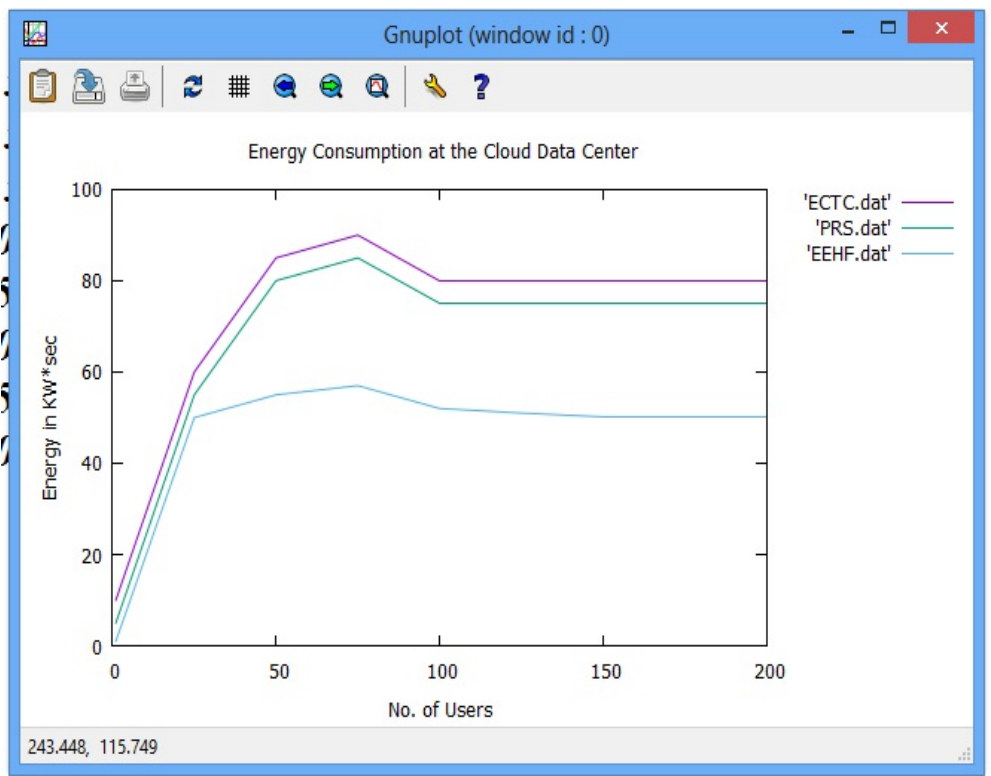

Fig. 4. Energy Consumption at the Cloud Data Center 


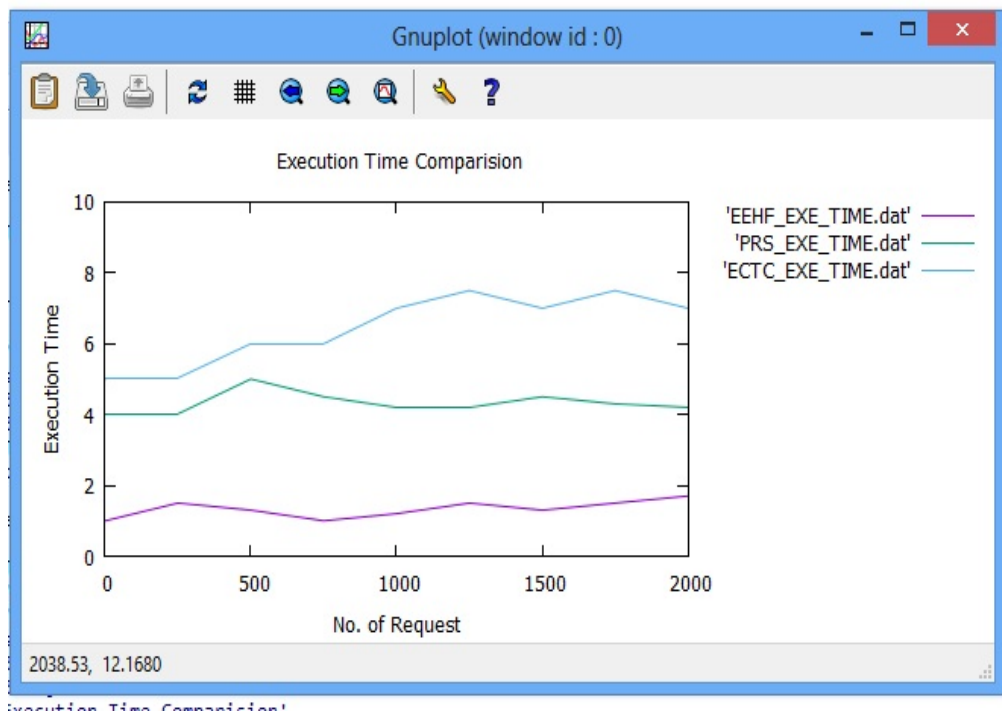

Fig. 5. \% of Execution Time Comparison the Cloud Data Centre

\section{Conclusion and Future Work}

Since we calculated the powerful upper edge rate by determining the standard supreme deviation and IQR of past data individually, the energy consumption on account of our carried out EEHF-based calculation is lower as compared to IQR(Interquartile Range) and irregular Virtual Machine VMs movement. We also set the upper edge usage of each PM by dissecting the entomb quartile scope of previous data one by one. As a result, the developer of the entomb quartile range (IQR) method only estimated the PM's CPU usage to find the overburden PM but did not pass judgment on the switches' power consumption at the cloud server farm. Furthermore, we moved the Virtual Machine VMs from not used PMs to used PMs due to an ad hoc Virtual Machine VMs relocation strategy, and as a result, we did not choose the most appropriate PMs for the Virtual Machine VMs relocation. As a result, PMs' power consumption is higher due to the possibility of Virtual Machine VMs being relocated. Furthermore, when comparing the proposed EEHF Virtual Machine VMs movement technique to the bury quartile range IQR and arbitrary Virtual Machine VMs relocation strategy as illustrated in, the proposed EEHF Virtual Machine VMs movement technique uses fewer resources. The explanation for the increased resource usage is due to our successfully implemented Virtual Machine VMs movement strategy. For the delivery of Virtual Machine VMs at the cloud server farm, we used fewer PMs and switches. As a result, there are fewer resources wasted at the cloud server farm.

\section{References}

[1] P. Barham, B. Dragovic, K. Fraser, S. Hand, T. Harris, A. Ho, R. Neugebauer, I. Pratt, and A. Warfield, "Xen and the specialty of virtualization," SIGOPS Oper. Syst. Fire up., vol. 37, no. 5, pp. 164-177, Oct. $2003 . \quad$ [Online]. Accessible:http://doi.acm.org/10.1145/1165389.945462

[2] N. K. Sharma and G. R. M. Reddy, "A tale energy-efficient resource distribution utilizing the hybrid methodology of hereditary dvfs with receptacle pressing," in 2015 Fifth International Conference on Communication Systems and Network Technologies, April 2015, pp. 111-115.

[3] "Novel energy-efficient virtual machine distribution at server farm utilizing hereditary calculation," in 2015 third International Conference on Signal Processing, Communication and Networking (ICSCN), March 2015, pp. 1-6.

[4] D. Kliazovich, P. Bouvry, and S. U. Khan, "Sanctums: server farm https://doi.org/10.1007/s10586-011-0177-4

[5] N. K. Sharma and G. R. M. Reddy, "A tale energy-efficient resource distribution utilizing the hybrid methodology of hereditary dvfs with container pressing," in 2015 Fifth International Conference on Communication Systems and Network Technologies, April 2015, pp. 111-115.

[6] N. K. Sharma and R. M. R. Guddeti, "Multi-target resources assignment utilizing improved hereditary calculation at the cloud server farm," in 2016 IEEE International Conference on Cloud Computing in Emerging Markets (CCEM), Oct 2016,pp.73-77.

[7] "On request virtual machine portion and movement at cloud server farm utilizing hybrid of feline multitude improvement and hereditary calculation," in 2016 Fifth International Conference on Eco-accommodating Computing and Communication Systems (ICECCS), Dec 2016, pp. 27-32.

[8] R. Poli, J. Kennedy, and T. Blackwell, "Molecule swarm streamlining," Swarm Intelligence, vol. 1, no. 1, pp. 33-57, Jun 2007. [Online]. Accessible: https://doi.org/10.1007/s11721-007-0002.

[9] L. Nieuwenhuis, M. Ehrenhardc and L. Prausec, "The shift to Cloud Computing: The effect of problematic innovation on the undertaking programming business biological system," Technological Forecasting and Social Change, Vol. 129, 2018, pp. 308-313.

[10] M. H. Ghahramani et al., "Toward Cloud Computing QoS Architecture: Analysis of Cloud Systems and Cloud Services," IEEE/CAA Journal of Automatica Sinica, Vol. 4, No. 1, Jan. 2017, pp. 5-17 
[11] Chen Li, Lisu Huo, and Huangke Chen, "Constant Workflows Oriented Hybrid Scheduling Approach with Balancing Host Weighted Square Frequencies in Clouds" IEEE Access, DOI: 10.1109/ACCESS.2019.2955013

[12] H. Yuan et al, "Biobjective Task Scheduling for Distributed Green Data Centers," IEEE Transactions on Automation Science and Engineering. doi: 10.1109/TASE.2019.2958979, online Jan.2020.

[13] H. Yuan, et al., "Spatial Task Scheduling for Cost Minimization in Distributed Green Cloud Data Centers," IEEE Transactions on Automation Science and Engineering, Vol. 16, No. 2, April 2019, pp. 729-740.

[14] H. Yuan and H. Liu, "Income and Energy Cost-streamlined Biobjective Task Scheduling for Green Cloud Data Centers," IEEE Transactions on Automation Science and Engineering, DOI: 10.1109/TASE.2020.2971512, on the web, Jan. 2020.

[15] D. Jiang et al, "An improvement based powerful directing calculation to energy-efficient organizations for distributed computing," Telecommunication Systems, 2016, Vol. 63, Issue 1, pp. 89-98. 